\title{
AN INTELLIGENT SYSTEM FOR THE ENHANCEMENT OF VISUALLY IMPAIRED NAVIGATION AND DisAster AssistanCE USING GEO-BASED POSITIONING AND MACHINE LEARNING
}

\author{
Wenhua Liang ${ }^{1}$, Ishmael Rico ${ }^{2}$ and Yu Sun ${ }^{3}$ \\ ${ }^{1}$ St.Margaret's Episcopal School, San Juan Capistrano, CA 92675 \\ ${ }^{2}$ University of California, Berkeley, CA, 94720 \\ ${ }^{3}$ California State Polytechnic University, Pomona, CA, 91768
}

\begin{abstract}
Technological advancement has brought the masses unprecedented convenience, but unnoticed by many, a population neglected through the age of technology has been the visually impaired population. The visually impaired population has grown through ages with as much desire as everyone else to adventure but lack the confidence and support to do so. Time has transported society to a new phase condensed in big data, but to the visually impaired population, this quick-pace living lifestyle, along with the unpredictable nature of natural disaster and COVID-19 pandemic, has dropped them deeper into a feeling of disconnection from the society. Our application uses the global positioning system to support the visually impaired in independent navigation, alerts them in face of natural disasters, and reminds them to sanitize their devices during the COVID-19 pandemic.
\end{abstract}

\section{KEYWORDS}

Geo-based navigation assistance, machine learning, mobile computing

\section{INTRODUCTION}

The advancement of technology has long been a significant accomplishment or representation of progress made for the convenience of the entire human race. However, as technologies continue to evolve for the fancy and quick-paced future that we all anticipate to witness, inconveniences are left behind to some neglected populations like the elderly population and the population with disabilities. Indeed, while technologies have contributed to the well-being of the mass and revolutionized the future, it would be unfair to ignore how it has also widened the technological generation gap, where the elderly population falls behind to adopt to new technologies, and the capacity-ability gap, where the population with disabilities feel challenged in face of technologies

[1]. According to the Centers for Disease Control and Prevention's Disability impacts All of Us infographic, 61 million adults in the United States live with some type of disability, which is, in fact, 1 in every 4 adults in the United States [2]. Among them, about 3 million experience blindness and difficulty with seeing. Even worse, as technologies enhance in this rapidly evolving world, problems have, otherwise, been introduced to the visually impaired population, and a list of such includes difficulty with navigation, communication, and the maintenance of a sense of 
independence by the visually impaired population in the ever-changing city. A research conducted by Yan Wu's team through questionnaires shows that while as much as $77 \%$ of visually impaired survey participants appreciate the value provided by technologies, only $23 \%$ of responders own smartphones (due to restricted accessibility and insufficient financial supports) compared to the $76 \%$ of sighted UK adults who own their own smartphones [4].

This clearly indicates that despite the immense technological advancement in this society, the visually impaired population experience inadequately from this advancement. In fact, many lack experience in even the very least of what are known to be successful technologies. Therefore, to respond to the World Health Organization's call to leave no one behind, our application aims to guide the visually impaired population toward independence [3].

Some common systems implemented for the guidance of visually impaired in navigation are the mobility tools like white canes and the guide dogs. The visually impaired people usually use the white cane to feel the changes on the surface of the pathway ahead of their steps. By swinging the cane from one side to another in rhythm with their feet, the users will be able to find and be aware of the obstructions ahead of them [5]. However, the effectiveness of the cane can be restricted when the cane breaks during a navigation, when poor weather or unfamiliar landmarks negatively influence its performance and, most importantly, when the person has to cross an intersection, considering that the white cane remains ineffective toward detecting vehicles in motion. In contrast, the guide dog, a trained pet for leading the visually impaired around obstacles, is aware of the danger imposed by the speeding vehicles and is thus a safer choice when it comes to crossing intersections. Indeed, guide dog is preferred by many for its mobility and companionship [6]. However, a guide dog can be an unconvincing choice for many because it requires additional cares, training, and expenses on goods to feed it. While guide dog and white cane are the most common systems employed by the visually impaired, they only provide assistance in independent navigation but lack assistance in other forms. Another proposed system that has helped the visually impaired to achieve independence is the subscription to real-time agents in needs of assistance. The idea behind this is that the visually impaired population can receive assistance immediately through a touch of a button or a call through applications on smartphone or laptop. While this definitely provides more forms of assistance to the users other than on independent navigation, the expenses are usually higher, and the assistance would not be there to be accompanied with the user twenty-four-seven. In fact, some platforms would not work without internet access, providing inconveniences and restrictions in navigation when the user travels to locations with no internet and thus access to support. Electronic glasses is another proposed system to help with not only the navigation of the user but in many other forms. In fact, its principle builds upon the capturing of real-time images through a camera and the displaying of these motions to the users' sight, which basically allows the users to live in a world with clear vision [7]. However, this piece of device is costly and not covered by medical insurances, so though many hope to experience vision with such advanced technology, the majority of them cannot afford it. Moreover, this device only works for those who are not completely blind, as it functions upon enhancing one's vision by displaying processed images.

In this paper, we follow the same line of research by assisting the visually impaired population to achieve a sense of independence through integration of software, hardware, and machinelearning. We have three goals to fulfill through this application:

1. Save data of the number of obstructions detected by the device in a one hour walk done by a visually impaired person (frequency) with its corresponding longitude and latitude into a database. With such database and through machine-learning, we will be able to predict frequency at similar categorizes of location using the characteristics of the specific location 
and to understand the relationship between frequency and different interior layout better for designing the most traveling-friendly layout for the visually- impaired in the future.

2. Use frequency, longitude, and latitude to determine whether the user is indoor (in an enclosed space) or outdoor (at an open space) so that instructions can be sent to the users who are at an unsafe type of location, either an enclosed space or an open space, during the specific natural disaster.

3. When reached a specific frequency, the user will be alerted to sanitize the device to avoid potential contact with Covid-19.

Our method is inspired by our aims to understand what's best for the visually impaired population but to also support this community during natural disasters and the COVID-19 pandemic. There are some significant features on our application. First, our application is voiced by computer instead of human agents, allowing for a greater scale of flexibility and accessibility everywhere at any time. Second, our application covers functions outside of independent navigation like an alerting system during natural disasters, which contributes toward the independence of the visually impaired through different aspects of their life. Third, as a technology itself, our device acts as a potential bridge to close the capacity-ability gap between the visually impaired population and the society, and it also makes the positivity within the advancement of technology apparent to the visually impaired population. Therefore, we believe that this application will connect the visually impaired population closer to the society and encourage them to be more independent and confident.

Experiments are performed to calculate and compare accuracies for determining the most appropriate machine learning model through the implementation of support vector learning (svm) and regression models. In the experiment, we tested different models through adjusting the regression model, polynomial parameters, and inputted data sets.

The rest of the paper is arranged as follows: Section 2 presents the essential background for this study. Section 3 provides the details on the challenges that the visually impaired population encounters; Section 4 proposes solutions in response to the challenges mentioned in Section 3; Section 5 discusses the relevant details about our process of experimentation, following by a presentation on related work in Section 6. Finally, Section 7 offers the conclusion remarks, as well as pointing out the future work of this project.

\section{BACKGROUND STUDY}

The section below illustrates current understanding and background information on cognitive mapping without visual experience and machine learning algorithms used in this study.

\subsection{Cognitive Mapping without Visual Experience}

It is most essential to have a comprehensive understanding on cognitive mapping without visual experience. The visual system has been concluded to be the most efficient in the obtention of spatial information for its ability to perform foveation along with peripheral vision - which haptic exploration does not support - and its outstanding performance in localization and object identification, which audial exploration does not support. That is, due to the proposal of such claim, questions have been raised on whether the visually impaired population is capable of obtaining a spatial understanding without experience with visual cues. Three major theories emerged and grew into debates as deficient theory, inefficiency theory, and the difference theory. The deficiency theory suggests that the lack of visual experience leads to the consequence of a 
total deficiency in spatial experience. On the other hand, the inefficiency theory and the difference theory suggest more positive outlooks. The inefficiency theory suggests the consequence of a similar but less efficient spatial experience in contrast to that of the sighted individuals while the difference theory suggests the consequence of a different but functionally equivalent experience. While evidences for both inefficiency theory and the difference theory exist in abundance, it is often suggested that the lack of visual experience does not prevent one from obtaining spatial knowledge because information from each of the different sense is specialized but also complementary and overlapping or in short, the visually impaired' $\mathrm{s}$ lack in experience with visual cues can be complemented by obtaining similar information from other senses though a bias in the system can be induced [16].

It is specifically inferred that as opposed to the deficiency theory, the poor performance by the congenitally blind compared to those who are sighted are usually due to factors like restricted capability in spatial processing or storage and the use of relatively less efficient information processing strategies. Many literature reviews and past studies on the same subject suggest a correlation in whether an individual is congenitally blind and the strategies implemented instinctively during navigation. For example, in an experiment involving the exploration of a largescale layout, the late blind and blindfolded sighted participants, who implemented the backandforth method, were able to perform better than the congenitally blind because their strategy allows the formation of a more integrated representation of a space. Such experiment supports that the processing strategy selected by the visually impaired - instead of their abilities - is what makes them less efficient than the sighted in navigation. That is, the visually impaired population usually choose sequential representation over map-like representation, which could be what impacted their performance in making spatial reference and caused them to be less efficient that the sighted individuals. In addition, the visually impaired has the tendency to map cognitively in reference to their own body over a reference that exists externally. The visually impaired individual prefers the strategy because it is the most reliable approach in existence for the obtention of spatial information under blind conditions, but if a task appears to be involved with mental reorganization, mental rotation or spatial inference, the approach becomes less effective in contrast to that utilized by the sighted individuals [17-19] .

\subsection{Machine Learning: SVM and Regression Algorithms}

Machine learning is commonly defined as computational methods that input and analyze existing data for performing the most efficient yet accurate prediction. It implements sets of machine learning algorithms to complete tasks by running through a representational process of training, validating, and testing data samples. The standard learning tasks implemented in this study are regression and support vector machines (SVMs) $[13,20]$.

\section{a. Support Vector Machines (SVMs)}

SVMs are machine learning technique developed to implement the characteristic of kernel for analyzing samples and classifying new profile into labels. In the use of SVM, a hyperplane is implemented between classifications in a high-dimensional space to analyze and separate existing samples. Such plane is selected to locate at a position where the adoption of maximum margin would be fulfilled so as to maximize accuracy in sample-based future predictions. Two characteristics- the soft margin and the kernel function- are also implemented within SVM to ensure the capture of the most data with adoption to minor misclassification. Specifically, the soft margin introduces a parameter that permits control over the extent of misclassification in algorithm while the kernel function allows computation with linear method in high-dimensional space for optimized simplicity. As the most essential in the technique of SVM, the kernel 
functions perform the major process of adding additional dimensions so as to better classify samples into labels [14]. While it may result in the overfit of data, kernel makes SVM competitive when compared to other machine learning techniques due to first, its capability to project data in higher dimensional space. Second, its capability to construct models of greater complexity in real-world application and, lastly, its capability to implement optimal simplicity so to achieve good performance in mathematical analyzation [15].

\section{b. Regression}

Regression is a machine learning technique implemented mathematically for the analysis of relationship between variables and for predicative purpose. Essentially, the technique measures the extent of effect one variable has on another for building an optimal model that best illustrates the dependency or independency between variables. Such technique is usually used for the two major theories of prediction and evaluation of casual relations between variables. The general definition for regression model is rather broad, as it points to all case where independent variable predict the dependent without precise direction that specifies on the quantities of variables put in use or the degree of polynomial implemented. To analyze in detail, the broad characterization of regression models can in fact be broken into smaller fragments, which would thus produce simple linear regression, multivariate linear regression (MLR), and polynomial regression. Despite their share in some common characteristics like the adoption to the least square method, these different regression models differ distinctively. The simple linear regression is inputted with only a single independent variable while MLR portray relationship for multiple independent variables. On the other hand, the polynomial regression differs significantly when compared to both MLR and simple linear regression in that it analyzes on a relationship modeled by nth degree polynomial while the former two regressions are modeled linearly $[21-22,26]$.

\subsection{Existing Systems}

A. Helal proposed a navigation system called Drishti for the visually impaired population through the integration of hardware like wearable computers and software built with a web of technological components such as spatial database and map server. Similar to our application, Drishti provides a significant amount of guidance to the user through voice support and addresses their needs through the active use of a digital map with GPS. Drishti readily involves the navigational features to support the visually impaired population, but our application extends support to disaster alert and the prevention of COVID-19 [11].

I.Ulrich proposed a navigation device called GuideCane to guide the visually impaired population during independent navigation. GuideCane is built with a cane on a wheel, and it implements a steering servo motor with an ultrasonic sensor for the main functionality to operate, which is to guide the users away from hazards and obstacles through the steering commands. While GuideCane performs its navigational feature on a wheel, our device operates on its own and is lighter and can be more portable for the users to bring everywhere for navigation [12].

T.Kiuru proposed a wearable mobility assistive device that implements radar technology. The operation of such device is based on an electromagnetic radar sensor that features at $24 \mathrm{GHz}$. During navigation, radio waves are transmitted and conveyed to the user by audial and/or haptic means to signal of the direction and distance of possible obstacles in front of the user. The device also features compensation for inclination errors through its ability to perform automatic inclination and orientation sensing. While the device is tested to be a potential for its portability, it is restricted to support in mobility [23]. 


\section{Challenges}

A few technical challenges have been identified and listed as follows. We will present the solution in Section 4.

\subsection{Challenge 1: Unsafe and Exclusive Urban Design}

In today's society, the layout of cities and the interior design of many buildings are planned and implemented to closely match with the major population's aesthetic perception and their desire toward a sense of freshness. While the aesthetic within these designs brings pleasure to a great majority, a building's exterior appearance in the society's vision has unreasonably dominated over the many other factors that should've been more significant or, to be specific, safety and convenience. To the many who are blessed with clear vision and have the ability to navigate freely in spaces, the domination of appearance over safety and convenience in designs does not seem to be a problem. However, to the visually impaired population who have nothing to rely upon when navigating in an unfamiliar space and only spatial memory to rely upon in a familiar space, a simple yet safe interior layout design saves them time and decreases their risk of injury. The unsafe and inconvenient city design has been the major factor which discourages the visually impaired population to enjoy the same quality of life and to travel freely around a city, so a solution has to be proposed to create a more friendly environment for the independent navigation of the visually impaired population.

\subsection{Challenge 2: Lack of Preparedness and Communication in Emergency Situation}

Humans are usually alerted about dangerous presences through their five senses - sight, hearing, smell, taste, and touch. During unexpected natural disasters, those nearest to the situation observe the danger to make judgement about what to do next while those who live farther away yet will possibly be impacted receive warnings on smart devices to prepare ahead of time. The visually impaired population, however, lack information to make judgments in face of the sudden onset of natural disasters or to prepare for such situations due to their vision loss, so a solution has to be proposed to take their community's safety into consideration during such crises.

\subsection{Challenge 3: Sanitization During and After the Pandemic}

The emergence of COVID-19, also known as the coronavirus disease, is a disease that leads to symptoms like dry cough, fever, and tiredness. Though this pandemic has introduced challenges to everyone, newly enforced rules like social distancing and the adjustments newly made to many markets have been particularly tough for the visually impaired population to adapt. [8] According to an article from The Conversation, a public media source in the United Kingdom, the changes in operation in markets has created difficulties for the visually impaired individuals to navigate using a spatial map and that social distancing has been difficult for them to employ in some situation due to their vision loss or inability to notice when people are walking overly close to them. [9] Moreover, the World Health Organization (WHO) mentions that the visually impaired population are potentially at a greater risk to contract COVID-19 due to their need to frequently touch items near them for directions during navigation. To limit the spread of COVID-19 in the visually impaired community and the whole society for a more immediate end to this pandemic, a solution must be proposed to guide the visually impaired population about the best method of navigation during this time and to develop procedures for limiting the chance of being exposed to COVID-19. 
International Journal of Grid Computing \& Applications (IJGCA) Vol.12, No.1/2/3, September 2021

\section{Solution}

\subsection{Overview to the Solution}

The application has been implemented using MIT App Inventor, and it is carefully developed to serve as a multi-functional platform to support the visually impaired population in navigations, during natural disasters, and in the midst of the COVID-19 pandemic. The application intends to take all aspects into consideration when it provides features like QR code login, locative marker placement, vibration when detected obstacles, alert in face of disasters, GPS-frequency database, and sanitization reminder.

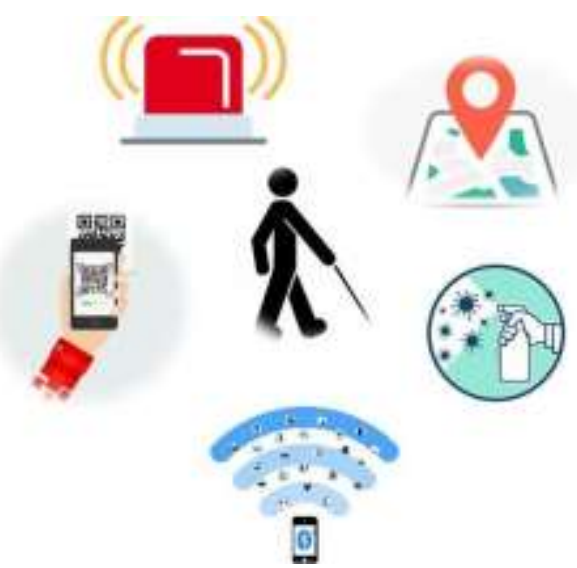

Figure 1: Overview of the solution 


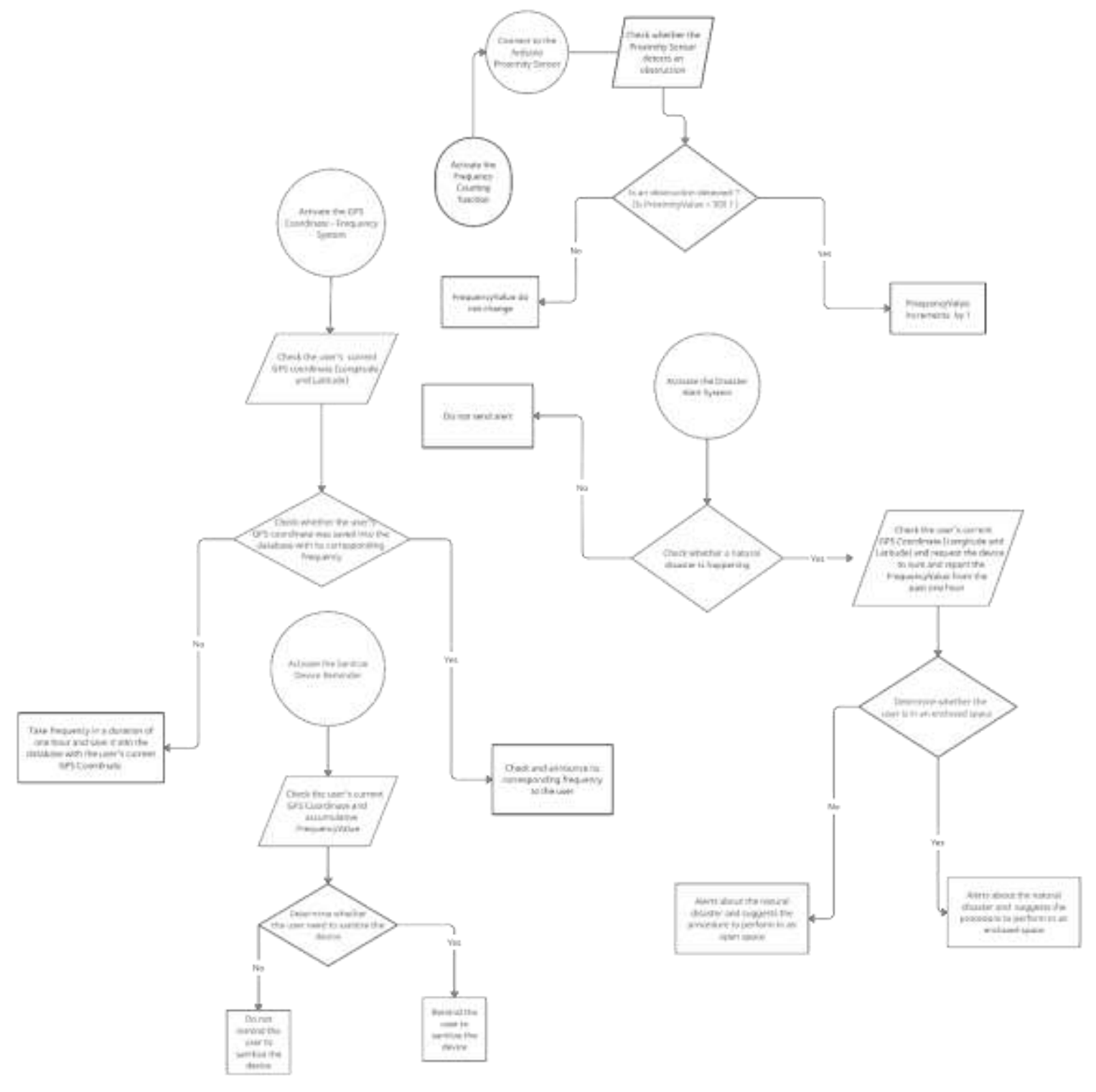

Figure 2: Overview of the solution (Flowchart)

\subsection{QR Code Login System}

Since the traditional login procedure of typing username and password can provide inconvenience to the visually impaired users and leads to reduced user experience, our application intends to prevent such inconvenience by allowing logins through QR codes. After the user signs up for an account, they can shake the smartphone to activate the QR code login system, which allows them to directly scan the code to login. The system also comes with voice guidance, which will provide feedback in response to successful login, allowing communication to happen directly between the application itself and the visually impaired users. 

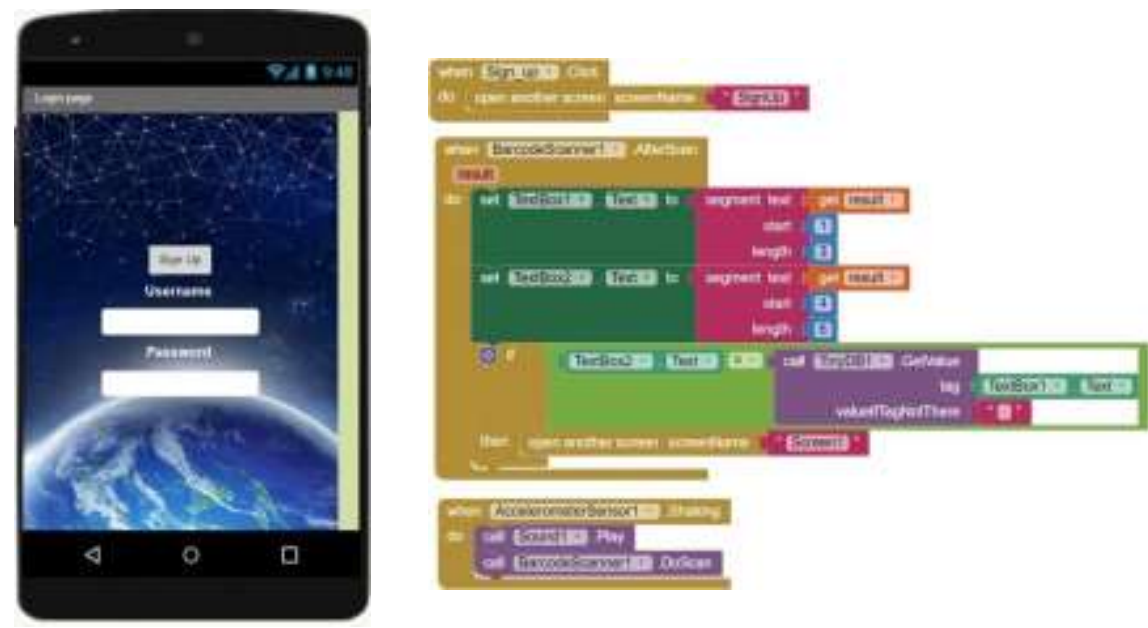

Figure 3: QR Code Login System

In our application, the Global Positioning System (GPS) is employed and used to display the user's current location on a map and in text. Longitude, latitude, and a detailed line of address are also displayed on screen after occurrences of new rounds of syncing. Through Bluetooth connection, this application can also connect to a smart cane which performs vital tasks like vibration in detection of obstructions and marker placement. The device -smart cane- itself is built using a proximity sensor, some wires, and a button from the Arduino kit. Shown in Figure 4, a proximity sensor is mounted near the tip of the smart cane to detect the surrounding or, specifically, the path before the user. Whenever the proximity sensor finds an obstruction that can distract and change the user's planned path for navigation, it will send a signal to the vibration motor to vibrate and through it, alert the user to change direction. Shown in Figure 2, the detection of an obstruction will also increment the frequency value displayed in the application by one, keeping a record of the frequency or how many times a visually impaired population would have its device detect an obstruction ahead of steps at different locations. Last but not least, the device has a button mounted on the very top. Whenever the button is pressed, a tiny red pin will be placed on the map. This can help the many users on the platform to mark and share locations that are inconvenient or unpleasant to navigate near at.
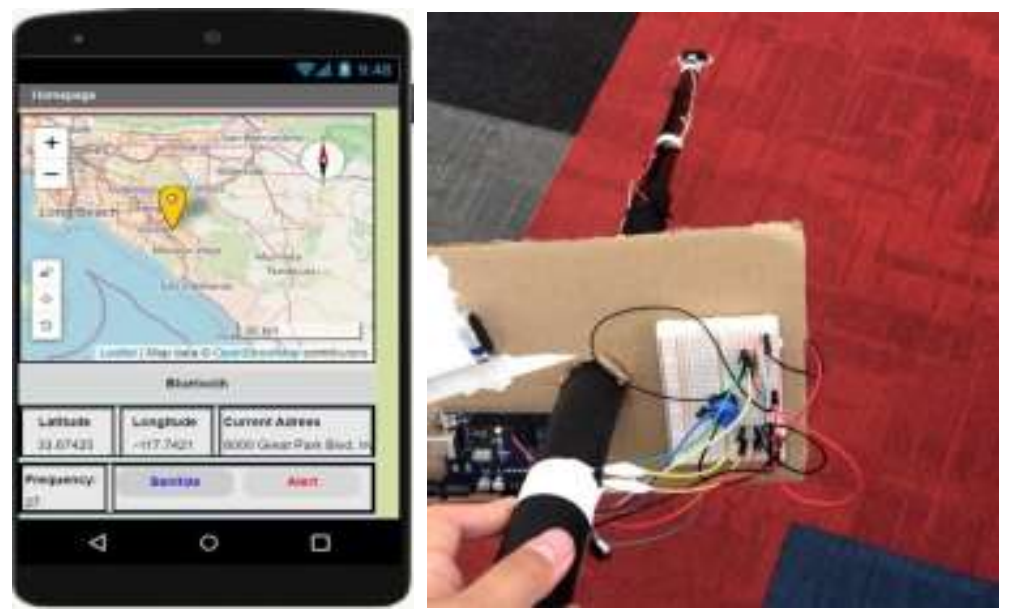

Figure 4: Global Positioning System 
International Journal of Grid Computing \& Applications (IJGCA) Vol.12, No.1/2/3, September 2021

\subsection{GPS Coordinate}

Our application encourages the users to navigate freely and, indeed, safely. With the global positioning system, the application accompanies the user on their road and requests consent to save their GPS coordinates with the device's corresponding acquired frequency at a specific location into a database. This will eventually produce a large database that can map out the locations that are more friendly for the visually impaired population to navigate nearby and, most importantly, raises the society's awareness toward making the environment more convenient and welcoming to every population, which would thus encourage entrepreneurs in the future to learn from those locations that succeed in making their spaces inclusive and accepting to everyone.

\subsection{Natural Disaster Alert System}

The frequency, along with the current longitude and latitude of the user, perform vital roles in the alert system for natural disasters. During a natural disaster, requests will be sent to the users' device to request for their data on frequency and GPS coordinate. With such information, services behind the calls will be able to make announcements and assign more personalized procedures to self-protect based on where the user is at and what resources are accessible near them.

\subsection{COVID-19 Sanitization Reminder}

Tracking frequency within the application also assists with the function of reminding the user to sanitize the device during the time of COVID-19 pandemic. Whenever a specific value of frequency is reached that the device has a high risk of having the virus on its surface, the sanitize button will light up on the application's home page while a voice will be played to remind the user to sanitize the device for minimizing the chance of contracting COVID-19.

\subsection{Machine-learning}

Dummy data were generated for machine learning in our program for testing and figuring out the most appropriate machine learning model through the use of svm and regression algorithms for the following three cases:

- use latitude, longitude, and the three categories of locations (home, store, park) to predict frequency.

- use longitude, latitude, and frequency to predict between the three categories of locations (home, store, park).

- use frequency to predict whether a user should sanitize the device.

\section{EXPERIMENT}

To evaluate the accuracy of the application's algorithms, we experimented with the different models, parameters, and data set features to find the most accurate machine learning model. The first experiment was conducted to find a machine learning model that best describes the relationship between the user's GPS Coordinates and the frequency of which an obstruction will be detected by the user's device in an one hour walk. This experiment evaluates and compares the accuracy of the three machine learning models created with 1000 datasets with different polynomial parameters. The second experiment, otherwise, is conducted with the intake of 1000 datasets with different set features to find a machine learning model that best predicts the user's presence in one of the three categories of locations (home, park, store). Lastly, the third 
experiment also consumes 1000 data sets with different set features for the purpose of predicting whether the user should sanitize their device at times. In each of the three experiments, the accuracy of different models is calculated and compared among each other to find the best representation in each that executes the most accurate prediction.

For experiment 1, machine learning models with different polynomial parameters were applied to the same data set to find out which model would produce the most accurate algorithm. A linear model, along with polynomial models with parameter 2,5 , and 7 , are compared together to evaluate the model that produces the most accuracy.

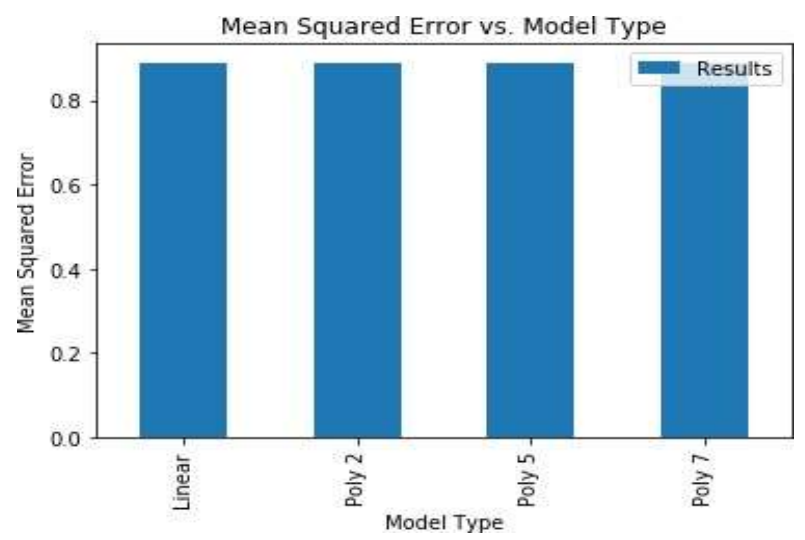

Figure 5: polynomial parameters influence on the model

The results of experiment 1 reveals neither being linear or polynomial nor changing the polynomial parameters influence the accuracy of the model. Shown in Figure 5, the accuracy of the linear model, along with that of the polynomial model with parameters of 2,5 , and 7 , are all at a value of 0.87 or a percentage of $87 \%$.

In experiment 2, three machine learning models that differ by their data sets are compared among each other for determining the one that is most accurate at predicting. The three models take in datasets of longitude, latitude, and frequency in different combinations, with the first model intaking three inputs (longitude, latitude, frequency), the second model intaking two inputs (longitude, latitude), and the third model intaking a single input (frequency).

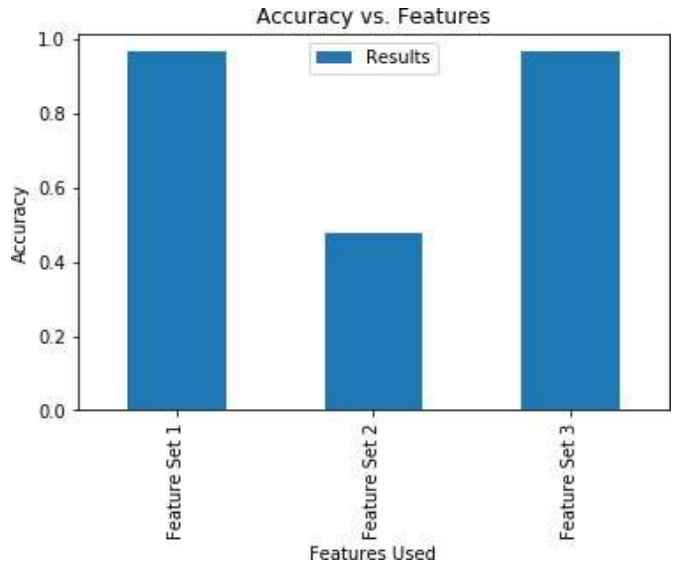

Figure 6: data sets influence on models

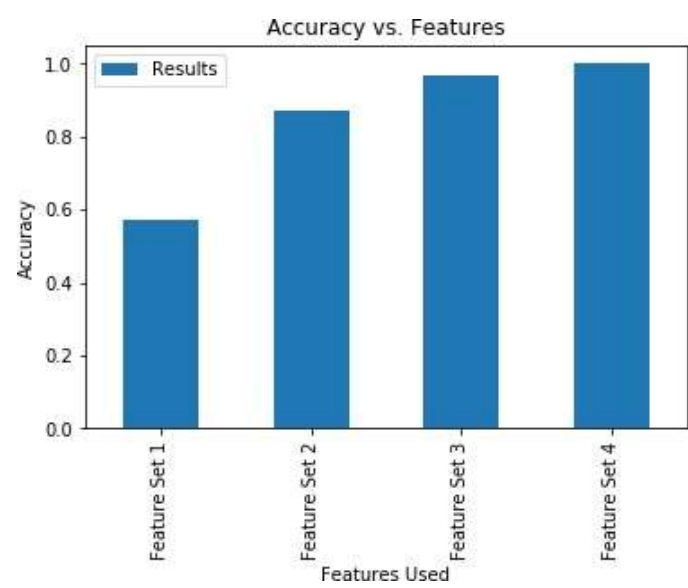

Figure 7: different data sets influence on four models 
The results of experiment 2 reveals that the accuracy for intaking the full data set which contains longitude, latitude, and frequency is lower than that when intaking a single dataset of frequency, as the accuracy for model 1 is shown to be at a value of 0.94 or a percentage of $94 \%$ while that of model 3 is a value of 0.95 or a percentage of $95 \%$. Amongst the three models, however, the model that intakes two inputs (longitude and latitude) differ the most and produces the least accuracy to a value of about 0.48 or $48 \%$. [Figure 6]

A total of four machine learning models with different data sets are compared against each other in experiment 3, in which the first model is created with two inputs (longitude, latitude), the second model with one single input of the three categories of location (home, park, or store), the third model with one single input of frequency, and the fourth with a full dataset of four inputs (longitude, latitude, frequency, categories of location).

The results of experiment 3 reveals that, like what happened in experiment 2, an increasing amount of intake dataset positively influences the accuracy of its model only in some cases. To support, Figure 7 displays the model with the most input (Figure 7 Feature Set 4) - longitude, latitude, frequency, and categories of location - as having the highest accuracy in a decimal of 0.98 and a percentage of $98 \%$. However, it is also significant to notice that the model producing the lowest accuracy is the one with intake of two inputs (Figure 7 Feature Set 1) instead of intake of one single input. To be specific, the model with longitude and latitude as the only inputs produces an accuracy in decimal of approximately 0.57 and in a percentage of $57 \%$, which is significantly lower than that produced by the models with one single intake in inputs or, to emphasize, an accuracy of greater than a value of 0.8 or percentage of $80 \%$. Lastly, the comparison of accuracy between model 2 and model 3 in Figure 7 emphasizes the difference in the accuracy of the same amount of input but different input or, in other words, between predicting based on a single input of the three categories of location from model 2 and based on a single input of frequency from model 3 . To compare, the machine learning model with frequency as its input has a higher accuracy in decimal of 0.96 or percentage of $96 \%$ than the machine model with categories of location as its single input, which has a lower accuracy of 0.84 in value and $84 \%$ in percentage. [Figure 7]

\begin{tabular}{|c|c|c|}
\hline & ML Techniques & Average Accuracy \\
\hline \multirow{4}{*}{ Experiment 1} & Linear & 0.87 \\
\hline & Degree 2 Polynomial & 0.87 \\
\hline & Degree 5 Polynomial & 0.87 \\
\hline & Degree 7 Polynomial & 0.87 \\
\hline \multirow{3}{*}{ Experiment 2} & SVM (with 3 sets: freq. ${ }^{1}$, Long. ${ }^{2}$, Lat. ${ }^{3}$ ) & 0.94 \\
\hline & SVM (with 2 sets: Long ${ }_{s, d}$ Lat.) & 0.48 \\
\hline & SVM (with 1 set: freq.) & 0.95 \\
\hline \multirow{4}{*}{ Experiment 3} & SVM (with 2 sets: Long, Lat.) & 0.57 \\
\hline & SVM (with 1 sets: satLoc ${ }^{4}$ ) & 0.84 \\
\hline & SVM (with 3 sets: freq. & 0.96 \\
\hline & 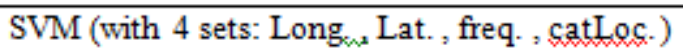 & 0.98 \\
\hline
\end{tabular}

Figure 8: Display of all results from experiment

\section{RELATED WORK}

J. M. Loomis emphasized the system of spatial display, including but not limited to body pointing, HPI-speech, virtual-tone display, and virtual-speech display, to be an effective system 
for navigational purpose of the visually-impaired population and proposed that virtual-speech display, regardless of its blockage toward environmental sounds, performs the best in leading to the fastest mean travel times and is rated subjectively as the top among the virtual displays used during the study [10].

Y. Zhang conducted a study to evaluate four regression models - which are standard Poisson regression, autoregressive adjusted Poisson regression, multiple linear regression, and seasonal autoregressive integrated moving average (SARIMA) model- and to identify the most appropriate one for modeling association between climate variation and salmonellosis transmission. The study was done through the comparison of parameter estimation, goodness-of-fit, and forecasting ability of the four models based on the notified salmonellosis cases in 2004. The study's result reports that though all models demonstrate consistent positive or negative association, the SARIMA model is the most appropriate to be adopted for the quantification of relationship between climate variations and salmonellosis transmission for its ability to integrate and control seasonal variation, autocorrection and long-term trend [24].

F. Laanaya studied and made comparison between a generalized additive model (GAM), logistic regression, residuals regression and linear regression to identify the one that best demonstrates the relationship between the dependent variable (average water temperature) and independent variables (daily average air temperature and average discharge at the Sainte-Marguerite River, Northern Canada). It is concluded in the end of the study through evaluation over each implementation's root mean square error (RMSE), bias error, Nash-Sutcliffe coefficient of efficiency (NSC), and goodness of fit that GAM performs the best due to its flexibility [25].

\section{CONClusion AND Future Work}

In conclusion, our application integrates with a Bluetooth-enabled device to encourage the visually impaired population to navigate independently, alert and guide them in natural disasters, and remind them to sanitize their devices during the pandemic. In our experiments, we tested trials of different machine learning models which differ by regression model, polynomial parameter, and inputted data sets. The first experimentation results show that since adjusting the regression model or polynomial parameter does not change the accuracy of the prediction at all, it's proper to just use a linear regression model to train and test for making predictions on frequency based on longitude, latitude, and the three categories of locations (home, park, store). The second and third experimentation results show that the machine learning model that intakes one single data set of frequency will predict among the three categories of locations the best and that the machine learning model that takes in the full data set of frequency, longitude, latitude, and the three categories of location will predict most accurately in whether or not to sanitize the device. The accuracy of each experiment when using the most appropriate machine learning model when making predictions all exists above $90 \%$ or a value of 0.90 , suggesting that the application will run well and accurately to ensure a pleasant and convenient experience for the visually impaired population.

While our application exceeds the navigational feature and remains unique from other prototypes in that it encourages independence in other aspects, it also has some limitations. Since the application interacts with the user solely through voice, it cannot provide the population that is both visually impaired and hearing-impaired the same quality of support. In the future, we plan to improve the accuracy of the system by boosting the signal connections between the device and API, strengthen the practicality by increasing the device portability, and enhance the optimization by adding a "help" button. We strongly believe that by following these measures, our alert system will be able to reach its full potential. 
International Journal of Grid Computing \& Applications (IJGCA) Vol.12, No.1/2/3, September 2021

\section{REFERENCES}

[1] Tracy L Mitzner, PhD, Jon A Sanford, March, Wendy A Rogers, PhD, Closing the CapacityAbility Gap: Using Technology to Support Aging with Disability, Innovation in Aging, Volume 2, Issue 1, January 2018, igy008, https://doi.org/10.1093/geroni/igy008

[2] “Disability Impacts All of Us Infographic." Centers for Disease Control and Prevention, Centers for $\begin{array}{llllll}\text { Disease Control and } & \text { Prevention, } & 16 & \text { Sept. } & \text { 2020, }\end{array}$ www.cdc.gov/ncbddd/disabilityandhealth/infographic-disability-impacts-all.html.

[3] “Assistive Technology." World Health Organization, World Health Organization, who.int/newsroom/fact-sheets/detail/assistive-technology.

[4] Wu, Y., Lindsay, S., Cable, J., Jones, R., Evans, L., \&Xie, X. (2018). Digital Media Usage of Sensory Impaired Users in Wales 2018 Report.

[5] Attia, I., \&Asamoah, D. (2020). The white cane. Its effectiveness, challenges and suggestions for effective use: The case of Akropong School for the Blind. Journal of Education, Society and Behavioural Science, 47-55.

[6] Whitmarsh, L. (2005). The benefits of guide dog ownership. Visual impairment research, 7(1), 27-42.

[7] Andrew Zaleski, special to CNBC.com. "Amazing Electronic Glasses Help the Legally Blind See, but They Are Costly." CNBC, CNBC, 20 Aug. 2018, www.cnbc.com/2017/09/20/theseamazingelectronic-glasses-help-the-legally-blind-see.html\#: :text=Amazing electronic glasses help the legally blind see, but they are costly,-Published Wed, Sep\&text=Smart glasses, called eSight3, help,price tag of about $\$ 10,000$.

[8] Senior Lecturer in Psychology. "The Pandemic Is Undermining Visually Impaired People's Independence - Here's How to Fix This.” The Conversation, 14 Jan. 2021, theconversation.com/thepandemic-is-undermining-visually-impaired-peoplesindependence-heres-how-to-fix-this-142209.

[9] “Coronavirus." World Health Organization, World Health Organization, www.who.int/healthtopics/coronavirus\#tab=tab_1.

[10] Loomis, J. M., Marston, J. R., Golledge, R. G., \&Klatzky, R. L. (2005). Personal guidance system for people with visual impairment: A comparison of spatial displays for route guidance. Journal of visual impairment \& blindness, 99(4), 219-232.

[11] Helal, A., Moore, S. E., \& Ramachandran, B. (2001, October). Drishti: An integrated navigation system for visually impaired and disabled. In Proceedings fifth international symposium on wearable computers (pp. 149-156). IEEE.

[12] Ulrich, I., \&Borenstein, J. (2001). The GuideCane-applying mobile robot technologies to assist the visually impaired. IEEE Transactions on Systems, Man, and Cybernetics-Part A: Systems and Humans, 31(2), 131-136.

[13] Mohri, M., Rostamizadeh, A., \& Talwalkar, A. (2018). Foundations of machine learning. MIT press.

[14] Noble, W. S. (2006). What is a support vector machine?.Nature biotechnology, 24(12), 15651567.

[15] Hearst, M. (Ed.), Dumais, S.T., Osuna, E. Platt, J. Scholkopf, B., Support Vector Machines, IEEE Intelligent Systems, Trends \& Controversies feature, 13 (4), July-August 1998.

[16] Millar, S. (1994-10-27). Understanding and Representing Space: Theory and Evidence from Studies with Blind and Sighted Children. : Oxford University Press. Retrieved 11 Jul. 2021

[17] Ungar, S. (2000). "Cognitive mapping without visual experience," in Cognitive Mapping. Past, Present, and Future, eds R. Kitchin and S. Freundschuh (London: Routledge), 221-248.

[18] Foulke, E. and Hatlen, P.H. (1992). A collaboration of two technologies. Part 1: Perceptual and cognitive processes: their implications for visually impaired persons. British Journal of Visual Impairment, 10, 43-46.

[19] Thinus-Blanc, C. and Gaunet, F. (1997) Representation of space in blind persons: vision as a spatial sense? Psychological Bulletin, 121, 20-42

[20] Shalev-Shwartz, S., \& Ben-David, S. (2014). Understanding Machine Learning: From Theory to Algorithms. Cambridge: Cambridge University Press. doi:10.1017/CBO9781107298019

[21] Maulud, D., \&Abdulazeez, A. M. (2020). A Review on Linear Regression Comprehensive in Machine Learning. Journal of Applied Science and Technology Trends, 1(4), 140-147.

[22] Ostertagová, E. (2012). Modelling using polynomial regression. Procedia Engineering, 48, 500-506.

[23] TeroKiuru, MikkoMetso, MikkoUtriainen, KirsimarjaMetsävainio, Hanna-Mari Jauhonen, RiittaRajala, ReetaSavenius, MirjaStröm, Tiia-Nina Jylhä, Reijo Juntunen\&JuhaSylberg | Duncan 
International Journal of Grid Computing \& Applications (IJGCA) Vol.12, No.1/2/3, September 2021

Shepherd (Reviewing Editor) (2018) Assistive device for orientation and mobility of the visually impaired based on millimeter wave radar technology-Clinical investigation results, Cogent Engineering, 5:1, DOI:10.1080/23311916.2018.1450322

[24] Zhang, Y., Bi, P., \& Hiller, J. (2008). Climate variations and salmonellosis transmission in Adelaide, South Australia: a comparison between regression models. International journal of biometeorology, 52(3), 179-187. https://doi.org/10.1007/s00484-007-0109-4

[25] Laanaya, F., St-Hilaire, A., \&Gloaguen, E. (2017). Water temperature modelling: comparison between the generalized additive model, logistic, residuals regression and linear regression models. Hydrological Sciences Journal, 62(7), 1078-1093.

[26] Lindley, D. (1991). Regression analysis: Theory, methods and applications, by A. Sen and M. Srivastava Pp 347. DM88. 1990. ISBN 0-387-97211-0 (Springer). The Mathematical Gazette,75(472), 248-249. doi:10.2307/3620306 